\title{
Prevalence of inadequate micronutrient intakes of Canadian long-term care residents
}

\author{
Heather H. Keller ${ }^{1,2 *}$, Christina Lengyel ${ }^{3}$, Natalie Carrier $^{4}$, Susan E. Slaughter ${ }^{5}$, Jill Morrison ${ }^{2}$, \\ Alison M. Duncan ${ }^{6}$, Catriona M. Steele ${ }^{7,8}$, Lisa Duizer ${ }^{9}$, K. Stephen Brown ${ }^{10}$, Habib Chaudhury ${ }^{11}$, \\ Minn N. Yoon ${ }^{12}$, Veronique Boscart ${ }^{1,13}$, George Heckman ${ }^{1}$ and Lita Villalon ${ }^{4}$ \\ ${ }^{1}$ Schlegel-University of Waterloo Research Institute for Aging, Waterloo, ON N2J OE2, Canada \\ ${ }^{2}$ Department of Kinesiology, University of Waterloo, Waterloo, ON N2L 3G1, Canada \\ ${ }^{3}$ Faculty of Agricultural \& Food Sciences, University of Manitoba, Winnipeg, MB R3T 2N2, Canada \\ ${ }^{4}$ École des sciences des aliments, de nutrition et d'études familiales, Faculté des sciences de la santé et des services \\ communautaires, Université de Moncton, Moncton, NB E1A 3E9, Canada \\ ${ }^{5}$ Faculty of Nursing, University of Alberta, Edmonton, AB T6G 1C9, Canada \\ ${ }^{6}$ Department of Human Health and Nutritional Sciences, University of Guelph, Guelph, ON N1G 2W1, Canada \\ ${ }^{7}$ Toronto Rehabilitation Institute, University Health Network, Toronto, ON, M5G 2A2, Canada \\ ${ }^{8}$ Rehabilitation Sciences Institute, Faculty of Medicine, University of Toronto, Toronto, ON M5G $1 \mathrm{~V} 7$, Canada \\ ${ }^{9}$ Department of Food Science, University of Guelph, Guelph, ON N1G 2W1, Canada \\ ${ }^{10}$ Department of Statistics and Actuarial Science, University of Waterloo, Waterloo, ON N2L $3 G 1$ Canada \\ ${ }^{11}$ Department of Gerontology, Simon Fraser University, Vancouver, BC V6B 5K3, Canada \\ ${ }^{12}$ School of Dentistry, University of Alberta, Edmonton, AB T6G 1C9, Canada \\ ${ }^{13}$ Conestoga College, Schlegel Centre for Advancing Seniors Care, Kitchener, ON, N2G 4M4, Canada
}

(Submitted 27 June 2017 - Final revision received 14 December 2017 - Accepted 20 December 2017-First published online 15 February 2018)

\section{Abstract}

This study determines the prevalence of inadequate micronutrient intakes consumed by long-term care (LTC) residents. This cross-sectional study was completed in thirty-two LTC homes in four Canadian provinces. Weighed and estimated food and beverage intake were collected over 3 non-consecutive days from 632 randomly selected residents. Nutrient intakes were adjusted for intra-individual variation and compared with the Dietary Reference Intakes. Proportion of participants, stratified by sex and use of modified (MTF) or regular texture foods, with intakes below the Estimated Average Requirement (EAR) or Adequate Intake (AI), were identified. Numbers of participants that met these adequacy values with use of micronutrient supplements was determined. Mean age of males ( $n$ 197) was 85.2 (sD 7.6) years and females ( $n$ 435) was 87.4 (sD 7.8) years. In all, 33\% consumed MTF; 78.2\% (males) and 76.1\% (females) took at least one micronutrient pill. Participants on a MTF had lower intake for some nutrients (males $=4$; females $=8$ ), but also consumed a few nutrients in larger amounts than regular texture consumers (males $=4$; females $=1$ ). More than $50 \%$ of participants in both sexes and texture groups consumed inadequate amounts of folate, vitamins $\mathrm{B}_{6}, \mathrm{Ca}, \mathrm{Mg}$ and $\mathrm{Zn}$ (males only), with $>90 \%$ consuming amounts below the EAR/AI for vitamin $\mathrm{D}, \mathrm{E}, \mathrm{K}, \mathrm{Mg}$ (males only) and K. Vitamin D supplements resolved inadequate intakes for 50-70\% of participants. High proportions of LTC residents have intakes for nine of twenty nutrients examined below the EAR or AI. Strategies to improve intake specific to these nutrients are needed.

Key words: Dietary energy: Macronutrients: Nutrient adequacy: Long-term care

Poor food intake is common among older adults living in longterm care homes (LTC), yet our knowledge of actual food intake and adequacy with respect to Dietary Reference Intake (DRI) recommendations is limited ${ }^{(1-3)}$. Inadequate intake, including micronutrients, can lead to diverse functional changes that are detrimental to health and cognitive function ${ }^{(4,5)}$. Although

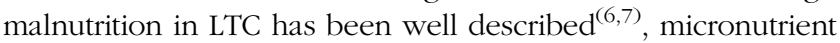
malnutrition is under-investigated. A first step towards

Abbreviations: AI, Adequate Intake; DRI, Dietary Reference Intake; EAR, Estimated Average Requirement; ICC, intra-class correlation; LTC, Long-term care home(s); M3, Making the Most of Mealtimes; ONS, oral nutritional supplement.

* Corresponding author: H. H. Keller, email hkeller@uwaterloo.ca 
improving micronutrient status is determining the micronutrient adequacy of the consumed diet. This evidence is necessary as the foundation for recommendations with respect to menu planning policies, raw food cost and adequate labour to produce nutrient-dense meals in LTC. Specifically, the distribution of resident nutrient intakes is required for menu planning at the home level to meet the $\mathrm{DRI}^{(8)}$ and prevent deficiencies.

Prior research on food intake is based on small, convenience samples ${ }^{(9-15)}$ with relatively inaccurate methods for collecting intake, including relying on staff to record consumption $^{(1,2,13,16)}$. Data on nutrient intake is not typically adjusted for intra-individual variation $^{(1,2,13)}$ nor is it based on a comprehensive panel of micronutrients ${ }^{(9,11,14,17,18)}$. Further, studies have yet to determine if current micronutrient supplement use mitigates an inadequate diet ${ }^{(1-3)}$ or if differences exist in nutrient consumption because of the requirement for a texture modified diet, despite the known increased vulnerability of these residents ${ }^{(19-21)}$. The Making the Most of Mealtimes (M3) prevalence study was designed to overcome these challenges and provide a better assessment of dietary intake in LTC. The purpose of this study was to determine the prevalence of inadequate micronutrient intakes consumed by LTC residents, comparing adequacy by diet texture and use of micronutrient supplements (e.g. pills).

\section{Methods}

\section{Sampling, subjects and recruitment}

Four provinces in Canada (Alberta, Manitoba, Ontario, New Brunswick) were included in the M3 study; eight LTC homes were purposively selected within each province to provide diversity in profit status, size, and cultural distinctiveness (e.g. predominately Jewish residents with Kosher meals). To be eligible for inclusion in this cross-sectional study, LTC had to have at least fifty residents who required more than $2 \mathrm{~h}$ of nursing care per day for basic activities of daily living, be in operation for more than 6 months and be supportive of the M3 data collection procedures. Within each LTC, two to four units were randomly selected for inclusion and if available, a unit dedicated to the care of residents with dementia was included. From these units, eligible residents were identified by trained home staff, that then used a random number list to approach residents who were interested in hearing more about the study. Up to forty interested, eligible and randomly identified residents were provided as a list to researchers who then completed informed written consent until a quota of twenty residents per home was reached. Inclusion criteria were as follows: medically stable (not recent or impending hospitalisation, not palliative), typically ate in the dining room, consumed food orally (residents with artificial feeding were excluded), were 65 years of age and older, and they or their alternative decision maker (ADM) provided informed consent to participate. Further details about the LTC, the protocol for recruitment and sample representativeness have been previously documented $^{(22)}$. Sample size estimation needed to take into account the provincial and home clusters and the intra-class correlation (ICC) of food intake among residents within the same facility (e.g. residents in the same home are more likely to have similar intakes) ${ }^{(22)}$. To estimate this ICC, previously published data on energy intake data from two Canadian studies involving several homes was attained and the ICC were calculated (mean $6322 \mathrm{~kJ}$ (1511 kcal), ICC 0.17; mean $4937 \mathrm{~kJ}(1180 \mathrm{kcal})$ ICC $0 \cdot 04)^{(22)}$. Using this range of estimated ICC, twenty residents in each of the thirty-two homes in the M3 sample provided sufficient statistical precision to estimate median energy intake with a $95 \%$ CI of $\pm 234-284 \mathrm{~kJ}$ $(56-68 \mathrm{kcal})$. Thus, twenty residents per home was considered a sufficient sample to estimate micronutrient intake in the M3 study.

\section{Data collection}

Only data collection pertinent to this analysis will be described; details of all procedures can be found in the protocol ${ }^{(22)}$. All food and beverage intake for 3 non-consecutive days ( 2 weekdays and 1 weekend day), at meals and between meals was recorded by trained research staff (two per province). In-person training was completed with each provincial coordinator who subsequently trained research assistants ${ }^{(22)}$. Food weigh scales (V22PWE3T; Ohaus) were identical among provinces and calibration was completed daily during data collection. Main plate food products were weighed before and waste weighed after consumption; any additional servings and condiments provided by staff were also recorded. Consumption of side dishes, snacks and beverage intake was estimated and before collection in each home, dishware was measured to determine volume. Use of salt at the table was not estimated. Morning and afternoon snack consumption was reported to researchers by residents, family, or staff and the evening snack and any other food consumed until midnight was recorded by staff. The complete menus with portion sizes and home recipes were provided to the researchers for nutrient analysis using the ESHA Food Processor Nutrition analysis software version 10.14.1 (ESHA Research). The Canadian Nutrient File was also used where appropriate for fortified and other foods specific to the Canadian food supply. Food and beverage intakes were entered into ESHA after each meal for each participant to ensure a thorough and accurate reporting and analysis; if required, LTC staff was asked to clarify ingredients or any substitutions that may have occurred. Where recipes were not available, researchers followed a standardised protocol to identify potential substitutes (e.g. other recipe from a home in the same province; recipe for a similar product from another province; or generic recipe from the internet based on product ingredients). Nutrient profiles of commercial products used were also accessed. Research assistants crosschecked data entry for accuracy and data were reviewed centrally to confirm completeness.

Before each meal, researchers also asked licensed nursing staff dispensing medications if prescribed oral nutritional supplements (ONS; e.g. commercial high protein/energy milkshakes) were provided and taken with medications; amount and type of ONS was recorded. Use of these nutritional supplements was included in the food/beverage estimation of 
nutrient intake. Micronutrient supplement (e.g. pill) use on the day of data collection was confirmed, with brands and type/ dose of nutrient recorded from the medication record. Only values from formulations that could be confirmed with confidence were used in this analysis (10.8\% not included: injectable nutrients, multivitamins where brand was not specified or any nutrient pill where dose was not recorded and/or could not reasonably be inferred (e.g. when unit was clearly recorded incorrectly)).

The medical record was also reviewed for each participant to determine their sex, birthdate, most current body weight, diet prescriptions (e.g. high-fibre diet, diabetic diet), prescribed diet texture (categorised using International Dysphagia Diet Standardisation Initiative (IDDSI) framework ${ }^{(23)}$ based on description in medical record and confirmation at meals as: regular, soft and bite sized, minced and moist, pureed, liquidised), diagnoses (total number and any dementia diagnosis), and use of prescription medication (total number). An ulna measurement ${ }^{(24)}$ was taken by the provincial project coordinator and used to estimate BMI. This coordinator also interviewed staff that worked with residents to complete key aspects from the LTC InterRAI, and specifically for this analysis the Cognitive Performance Scale (CPS) and the Activities of Daily Living scale (ADL). The CPS was used to describe resident cognitive status. It is a seven-point scale, where 0 represents no cognitive impairment and 6 represents very severe cognitive impairment with the person unable to make daily decisions, make themselves understood or eat independently ${ }^{(25)}$. The ADL score has a maximum of 28 and higher scores indicate increased dependency for various activities of daily living ${ }^{(26)}$. Finally, the Edinburgh Feeding Evaluation in Dementia Questionnaire (Ed-FED-Q), a valid measure of eating challenges at mealtimes, was collected by observation at three meals. Scores range from 10 to 30 and the average across the three observed meals was calculated for each resident, where higher scores indicate more eating challenges ${ }^{(27)}$

\section{Statistical analyses}

Only participants whose food intake was observed over at least six mealtimes (equivalent to $2 \mathrm{~d}$ ) were used in this analysis ( $n 632 ; 519(82.1 \%$ ) had all nine meals, $98(15.5 \%$ ) had seven to eight meals, and $15(2 \cdot 4 \%)$ had six meals). Participant characteristics were summarised (mean values and standard deviations, frequency) and compared by sex group and regular and modified textures using Student's $t$ test, or the $\chi^{2}$ test. A $P$ value for statistical significance was set at $<0.01$ due to the number of comparisons completed (Table 1 ; test statistics and $P$ values provided in text).

Food and fluid intake data were stratified by sex and diet texture for comparison with the DRI. Modified texture for this analysis was defined as those consuming a 'liquidised', 'pureed' or 'minced and moist' texture (IDDSI 3-5), whereas the regular category included 'soft and bite sized' (IDDSI 6) and 'regular' (IDDSI 7) consumers. To enable comparison of nutrient intakes to the $\mathrm{DRI}^{(28)}$ usual intake of nutrients was estimated by adjusting 3-d average intakes for intra-individual variation according to the method outlined by the National Research
Council $^{(28,29)}$. The first comparison to the DRI was based on all food, beverage and ONS consumed. A second comparison to the DRI was completed, with micronutrients from micronutrient pills added to the adjusted food/beverage/ONS intake values for those participants consuming these preparations. Percentiles of the distribution of intake were determined for each sex and for regular and modified texture diet groups to determine prevalence of inadequacy. Median, quartile 1 (Q1) and quartile 3 (Q3) values are reported. The distribution of nutrient intake from food/beverage/ONS was used to determine the proportion of participants with inadequate intake for those nutrients with an Estimated Average Requirement (EAR) using the EAR cut-point method ${ }^{(28)}$. For nutrients with an Adequate Intake ( $\mathrm{AI}$; vitamin $\mathrm{K}, \mathrm{K}, \mathrm{Na}$ ), the proportion of participants falling below the AI with respect to their intake is reported. The number of participants, whose intake surpassed the EAR/AI with micronutrient supplements, is also reported. Median intake and proportion below the EAR/AI were compared for modified texture and regular texture consumers using Wilcoxon Mann Whitney and chi-square tests. All analyses were performed using SAS $^{\circledR}$ software package version 9.4 (SAS Institute)

\section{Ethics}

All study participants or alternative decision makers for residents provided their written consent to participate. This protocol received clearance from ethics boards at the University of Waterloo, University of Alberta (Pro00050002), University of Manitoba (J2014:139), Université de Moncton (1415-022) and University Hospital Network, University of Toronto (16-5051-DE). Some individual LTC homes also required ethics review by a local/regional committee.

\section{Results}

Participant characteristics are provided in Table 1. Modified texture consumers were $33 \%$ (208/632) of the sample. Female participants consuming modified texture foods had a lower BMI than women consuming regular texture foods and women had lower average BMI than men (women's mean: 22.7 (SD 4.8$) \mathrm{kg} / \mathrm{m}^{2}$; male mean: $26 \cdot 2$ (sD 6$) \mathrm{kg} / \mathrm{m}^{2} \cdot 12 ; t=6.38, P<0.001$ ). In females, protein intake in $\mathrm{g} / \mathrm{kg}$ body weight was significantly higher and energy intake significantly lower for those consuming a modified texture as compared with those consuming a regular texture (energy: $t=2.95, \quad P=0.004$; protein: $t=-4.39$, $P<0.001)$. The proportion of participants with dementia was significantly higher for females consuming a modified texture $(81.2 \%)$ as compared with females consuming regular texture foods (59.6\%; $\chi^{2}=19 \cdot 65, P<0 \cdot 001$; OR $2 \cdot 92 ; 95 \%$ CI $\left.1 \cdot 8,4.75\right)$. CPS scores were higher (greater cognitive impairment) across both sexes for those consuming modified texture foods as compared with those consuming regular texture foods (male: $t=-5.53, P<0.001$; female: $t=-11.26, P<0.001)$. Similarly, higher ADL (more dependency) and Ed-FED-Q (more eating challenges) were seen in those consuming modified texture foods as compared with those consuming regular texture foods 
Table 1. Making the Most of Mealtimes sample description and comparisons by sex and food texture (Mean values and standard deviations; percentages; $n$ 632)

\begin{tabular}{|c|c|c|c|c|c|c|}
\hline & \multicolumn{3}{|c|}{ Male } & \multicolumn{3}{|c|}{ Female } \\
\hline & \multirow[b]{2}{*}{ All } & \multicolumn{2}{|c|}{ Diet textureł } & \multirow[b]{2}{*}{ All } & \multicolumn{2}{|c|}{ Diet textureł } \\
\hline & & Regular & Modified & & Regular & Modified \\
\hline \multicolumn{7}{|l|}{ Demographics } \\
\hline$n$ & 197 & 127 & 70 & 435 & 297 & 138 \\
\hline \multicolumn{7}{|l|}{ Age (years) } \\
\hline Mean & $85 \cdot 2^{*}$ & 84.9 & $85 \cdot 7$ & 87.4 & $87 \cdot 3$ & $87 \cdot 8$ \\
\hline SD & 7.6 & 7.8 & 7.4 & $7 \cdot 8$ & 8.0 & 7.5 \\
\hline \multicolumn{7}{|l|}{ BMI $\left(\mathrm{kg} / \mathrm{m}^{2}\right) \S$} \\
\hline Mean & $26 \cdot 0^{*}$ & $26 \cdot 6$ & $24 \cdot 8$ & $25 \cdot 1$ & $26 \cdot 2$ & $22 \cdot 7 \dagger$ \\
\hline \multirow{2}{*}{\multicolumn{7}{|c|}{ Diagnoses and challenges }} \\
\hline & & & & & & \\
\hline \multicolumn{7}{|l|}{ Total number of diagnoses } \\
\hline Mean & $5 \cdot 3$ & 5.4 & $5 \cdot 1$ & 5.4 & 5.5 & $5 \cdot 3$ \\
\hline SD & $2 \cdot 0$ & 2.0 & $2 \cdot 1$ & 2.0 & $2 \cdot 1$ & 1.9 \\
\hline Dementia diagnosis (\%) & 61.9 & 60.6 & $64 \cdot 3$ & $66 \cdot 4$ & $59 \cdot 6$ & $81 \cdot 2 \dagger$ \\
\hline \multicolumn{7}{|l|}{ Number of medications prescribed (\%) } \\
\hline $0-4$ drugs & $16 \cdot 2$ & $12 \cdot 6$ & $22 \cdot 9$ & $23 \cdot 7$ & 21.9 & $27 \cdot 5$ \\
\hline $5-9$ drugs & 53.3 & 55.1 & $50 \cdot 0$ & $49 \cdot 4$ & $47 \cdot 8$ & $52 \cdot 9$ \\
\hline & 30.5 & $32 \cdot 3$ & $27 \cdot 1$ & $26 \cdot 9$ & 30.3 & $19 \cdot 6$ \\
\hline \multicolumn{7}{|l|}{$\begin{array}{l}\geq 10 \text { drugs } \\
\text { Cognitive Performance Scale§ }\end{array}$} \\
\hline Mean score (max. 6) & $2 \cdot 6^{*}$ & $2 \cdot 2$ & $3.5 \dagger$ & 3.0 & $2 \cdot 4$ & $4.3 \dagger$ \\
\hline SD & 1.6 & 1.4 & 1.7 & 1.8 & 1.6 & 1.7 \\
\hline$\%$ Moderate/severe impairment (score $\geq 3$ ) & 51.8 & 40.9 & $71.4 \dagger$ & $57 \cdot 2$ & $45 \cdot 2$ & $83 \cdot 1 \dagger$ \\
\hline \multicolumn{7}{|l|}{ ADL Score $(\max .28) \S$} \\
\hline Mean & 14.5 & $12 \cdot 1$ & $18 \cdot 8 \dagger$ & $15 \cdot 2$ & $12 \cdot 7$ & $20.6 \dagger$ \\
\hline SD & $7 \cdot 7$ & $7 \cdot 0$ & $7 \cdot 1$ & 7.9 & $7 \cdot 3$ & $6 \cdot 2$ \\
\hline Ed-FED-Q score & & & & & & \\
\hline Mean & $12 \cdot 0$ & $11 \cdot 3$ & $13 \cdot 3 \dagger$ & 12.5 & 11.8 & $14.0 \dagger$ \\
\hline SD & $2 \cdot 2$ & 1.3 & $2 \cdot 7$ & $2 \cdot 3$ & 1.7 & $2 \cdot 6$ \\
\hline Diet and nutrition & & & & & & \\
\hline ONS prescribed (\%) & 30.5 & $21 \cdot 3$ & $47 \cdot 1 \dagger$ & $30 \cdot 6$ & 20.5 & $52 \cdot 2 \dagger$ \\
\hline Number of micronutrient supplements prescrib & & & & & & \\
\hline Mean & 1.5 & 1.7 & $1 \cdot 1 \dagger$ & 1.5 & 1.6 & $1.2 \dagger$ \\
\hline SD & 1.2 & 1.3 & 1.0 & $1 \cdot 2$ & 1.1 & 1.3 \\
\hline Number of micronutrients prescribed (\%) & & & & & & \\
\hline None & 21.8 & $17 \cdot 3$ & 30.0 & 23.9 & 18.2 & $36 \cdot 2$ \\
\hline 1-3 prescribed & 71.1 & $73 \cdot 2$ & $67 \cdot 1$ & 69.9 & $75 \cdot 8$ & $57 \cdot 2$ \\
\hline 4-6 prescribed & $7 \cdot 1$ & $9 \cdot 4$ & 2.9 & $6 \cdot 2$ & $6 \cdot 1$ & $6.5 \dagger$ \\
\hline Single nutrient supplements prescribed (\%) & 64.5 & 65.4 & $62 \cdot 8$ & $60 \cdot 7$ & 64.6 & $52 \cdot 2$ \\
\hline Multivitamin prescribed (\%) & 13.7 & $17 \cdot 3$ & $7 \cdot 1$ & $15 \cdot 4$ & $17 \cdot 2$ & 11.6 \\
\hline IDDSI diet texture (\%) & & & & & & \\
\hline Regular & 51.8 & $80 \cdot 3$ & - & $53 \cdot 3$ & $78 \cdot 1$ & - \\
\hline Soft and bite sized & $12 \cdot 7$ & $19 \cdot 7$ & - & 14.9 & 21.9 & - \\
\hline Minced and moist & $26 \cdot 9$ & - & $75 \cdot 7$ & 19.5 & - & 61.6 \\
\hline Pureed & 8.6 & - & $24 \cdot 3$ & 11.7 & - & $37 \cdot 0$ \\
\hline Liquidised & 0.0 & - & 0.0 & 0.46 & - & 1.4 \\
\hline Any diet prescription (\%) & $41 \cdot 1$ & 40.9 & 41.4 & $36 \cdot 3$ & 38.0 & 32.6 \\
\hline Energy intake $(\mathrm{kJ} / \mathrm{d})$ & & & & & & \\
\hline Mean & $7186 \cdot 4^{*}$ & $7276 \cdot 8$ & $7022 \cdot 8$ & $6189 \cdot 0$ & 6309.5 & $5935.8 \dagger$ \\
\hline SD & $1220 \cdot 0$ & 1251.8 & $1149 \cdot 8$ & $1106 \cdot 7$ & 974.4 & $1317 \cdot 1$ \\
\hline Energy intake (kcal/d) & & & & & & \\
\hline Mean & $1717 \cdot 6^{*}$ & $1739 \cdot 2$ & 1678.5 & $1479 \cdot 2$ & $1507 \cdot 3$ & $1418 \cdot 7 \dagger$ \\
\hline SD & 291.6 & 299.2 & 274.8 & 264.5 & 232.9 & 314.8 \\
\hline Protein intake (g/d) & & & & & & \\
\hline Mean & $64 \cdot 7^{*}$ & $65 \cdot 1$ & 63.9 & $54 \cdot 1$ & 54.2 & 54.0 \\
\hline SD & $12 \cdot 8$ & $12 \cdot 21$ & 13.8 & 11.8 & $10 \cdot 0$ & $15 \cdot 0$ \\
\hline Protein intake $(\mathrm{g} / \mathrm{kg}$ per $\mathrm{d}) \|$ & & & & & & \\
\hline Mean & 0.88 & 0.85 & 0.93 & 0.90 & 0.85 & $1.00 \dagger$ \\
\hline SD & 0.23 & 0.18 & 0.30 & 0.27 & 0.22 & 0.35 \\
\hline Fibre intake $(\mathrm{g} / \mathrm{d})$ & & & & & & \\
\hline Mean & $13.9^{*}$ & 14.5 & $12 \cdot 7 \dagger$ & 11.8 & $12 \cdot 2$ & $11.0 \dagger$ \\
\hline SD & $3 \cdot 2$ & 3.3 & $2 \cdot 4$ & 3.3 & 3.1 & 3.6 \\
\hline
\end{tabular}

ADL, activities of daily living; CPS, Cognitive Performance Scale; Ed-FED-Q, Edinburgh Feeding Evaluation in Dementia Questionnaire; ONS, oral nutritional supplement; IDDSI, International Dysphagia Diet Standardisation Initiative.

Values are significantly different between males and females: ${ }^{*} P<0.01$.

Values are significantly different between modified and regular texture categories, within sex group: $\dagger P<0.01$.

‡ Regular textures include 'regular' and 'soft and bite sized' (IDDSI categories 7 and 6); modified textures include 'minced and moist', 'pureed' and 'liquidised' (IDDSI 5, 4 and 3).

$\S$ Missing values: ADL Score, $n$ 627; BMI, $n$ 620; CPS score, $n 627$.

|| $\mathrm{kg}$ of body weight. 
for both sexes. Those consuming modified textures for both sex groups were more likely to receive ONS than those consuming regular textures (male: $\chi^{2}=14.27, P<0.001$; OR 3.3; $95 \% \mathrm{CI}$ 1.75, 6.22; female: $\chi^{2}=44.42, P<0.001$; OR $4.22 ; 95 \%$ CI 2.73 , 6.53). Micronutrient supplement use was significantly different across texture comparisons for both sexes with those consuming regular textures being prescribed more vitamin/minerals than those consuming modified textures (male: $t=3 \cdot 71$, $P<0.001$; female: $t=3 \cdot 20, P=0.002)$. The online Supplementary Table $\mathrm{S} 1$ provides a summary of the proportion of residents taking various micronutrient supplements and the dosage provided. About three-quarters of participants were consuming at least one formulation, with single nutrients being the most common including: vitamin $\mathrm{D}$, vitamin $\mathrm{B}_{12}, \mathrm{Ca}, \mathrm{Fe}$ and vitamin C.

Tables 2 and 3 provide median (Q1, Q3) micronutrient intakes from food/beverage/ONS intake for males and females, respectively, for those consuming regular or modified texture diets. For males (Table 2), median values for the following nutrients were significantly lower for modified textures $\mathrm{v}$. regular texture participants: folate, Fe, Se and Na. Vitamin C, D, Ca and $\mathrm{Zn}$ were consumed in higher amounts for modified texture

Table 2. Nutrient analysis of food intake only and food intake with micronutrient supplements added for males on regular ( $n$ 127) and modified ( $n$ 70) texture diets

\begin{tabular}{|c|c|c|c|c|c|c|c|c|c|c|c|}
\hline \multirow[b]{2}{*}{ Micronutrients } & \multirow[b]{2}{*}{ EAR } & \multirow[b]{2}{*}{$\begin{array}{c}\text { Diet } \\
\text { texture }\end{array}$} & \multicolumn{4}{|c|}{ Food intake only $\ddagger$} & \multicolumn{5}{|c|}{ Food intake and micronutrient supplements } \\
\hline & & & Q1 & Median & Q3 & $\begin{array}{l}\% \text { of individuals } \\
\text { below EAR/AI }\end{array}$ & $n \S$ & Q1 & Median & Q3 & $\begin{array}{c}\text { Number of individuals } \\
>\text { EAR because of } \\
\text { supplementation }\end{array}$ \\
\hline \multirow{2}{*}{ Vitamin A RAE $(\mu \mathrm{g})$} & \multirow{2}{*}{625} & Regular & $619 \cdot 6$ & 721.9 & $825 \cdot 9$ & $26 \cdot 8$ & 120 & 634.5 & $723 \cdot 6$ & $829 \cdot 0$ & 1 \\
\hline & & Modified & $594 \cdot 0$ & $724 \cdot 8$ & $970 \cdot 8$ & $30 \cdot 0$ & 67 & $590 \cdot 1$ & 718.9 & $927 \cdot 9$ & 0 \\
\hline \multirow{2}{*}{ Vitamin $B_{1}(\mathrm{mg})$} & \multirow[t]{2}{*}{1} & Regular & 1.2 & 1.4 & 1.6 & $7 \cdot 9$ & 119 & $1 \cdot 2$ & 1.4 & $1 \cdot 7$ & 0 \\
\hline & & Modified & $1 \cdot 2$ & 1.4 & $1 \cdot 6$ & $4 \cdot 3$ & 67 & $1 \cdot 2$ & 1.4 & 1.6 & 0 \\
\hline \multirow{2}{*}{ Vitamin $B_{2}(\mathrm{mg})$} & \multirow{2}{*}{$1 \cdot 1$} & Regular & $1 \cdot 8$ & $2 \cdot 0$ & 2.5 & 2.4 & 119 & $1 \cdot 8$ & $2 \cdot 1$ & 2.5 & 0 \\
\hline & & Modified & 1.7 & $2 \cdot 0$ & $2 \cdot 3$ & $7 \cdot 1$ & 67 & 1.7 & $2 \cdot 0$ & $2 \cdot 3$ & 0 \\
\hline \multirow[t]{2}{*}{ Niacin (mg) } & \multirow[t]{2}{*}{12} & Regular & $23 \cdot 1$ & $26 \cdot 4$ & 30.5 & 0.8 & 124 & $22 \cdot 6$ & $26 \cdot 3$ & 30.5 & 0 \\
\hline & & Modified & $21 \cdot 3$ & 24.9 & $29 \cdot 0$ & 1.4 & 70 & $21 \cdot 3$ & $24 \cdot 9$ & $29 \cdot 0$ & 0 \\
\hline \multirow[t]{2}{*}{ Vitamin $B_{6}(\mathrm{mg})$} & \multirow[t]{2}{*}{1.4} & Regular & $1 \cdot 1$ & $1 \cdot 3$ & 1.5 & $61 \cdot 4$ & 118 & $1 \cdot 1$ & 1.4 & 1.5 & 2 \\
\hline & & Modified & 1.2 & 1.4 & 1.6 & $60 \cdot 0$ & 67 & $1 \cdot 1$ & $1 \cdot 3$ & 1.6 & 0 \\
\hline \multirow[t]{2}{*}{ Folate DFE $(\mu \mathrm{g})$} & \multirow[t]{2}{*}{320} & Regular & $228 \cdot 8$ & $266 \cdot 6^{\star \star}$ & $335 \cdot 0$ & $73 \cdot 2 † \dagger$ & 122 & $231 \cdot 1$ & $272 \cdot 6^{\star \star}$ & $359 \cdot 4$ & 8 \\
\hline & & Modified & $177 \cdot 1$ & $219 \cdot 2$ & $257 \cdot 1$ & 91.4 & 68 & $176 \cdot 8$ & $219 \cdot 2$ & $256 \cdot 0$ & 1 \\
\hline \multirow{2}{*}{ Vitamin $B_{12}(\mu \mathrm{g})$} & \multirow[t]{2}{*}{2} & Regular & $3 \cdot 3$ & 3.9 & 4.6 & $3 \cdot 2$ & 119 & 3.6 & 4.6 & $504 \cdot 1$ & 1 \\
\hline & & Modified & $3 \cdot 4$ & $4 \cdot 2$ & $5 \cdot 1$ & $4 \cdot 3$ & 66 & $3 \cdot 7$ & $5 \cdot 1$ & $502 \cdot 8$ & 1 \\
\hline \multirow[t]{2}{*}{ Vitamin C (mg) } & \multirow[t]{2}{*}{75} & Regular & $61 \cdot 1$ & $90 \cdot 2^{\star \star}$ & 110.4 & $34.6 † \dagger$ & 116 & $64 \cdot 2$ & $92 \cdot 6^{\star *}$ & $124 \cdot 5$ & 6 \\
\hline & & Modified & $87 \cdot 7$ & $120 \cdot 0$ & $154 \cdot 8$ & $14 \cdot 3$ & 66 & $87 \cdot 7$ & $125 \cdot 4$ & $156 \cdot 9$ & 0 \\
\hline \multirow[t]{2}{*}{ Vitamin D $(\mu \mathrm{g})$} & 10 & Regular & 4.4 & $5 \cdot 6^{\star \star}$ & $7 \cdot 2$ & $97.6 \dagger$ & 124 & $7 \cdot 2$ & $31 \cdot 1$ & $50 \cdot 4$ & 83 \\
\hline & & Modified & $5 \cdot 4$ & $7 \cdot 1$ & $8 \cdot 7$ & $90 \cdot 0$ & 70 & 6.5 & $31 \cdot 1$ & 34.4 & 38 \\
\hline Vitamin E & 12 & Regular & 4.5 & $5 \cdot 4$ & $6 \cdot 2$ & 98.4 & 114 & 4.6 & $5 \cdot 7^{*}$ & $6 \cdot 8$ & 6 \\
\hline & & Modified & 3.9 & $4 \cdot 8$ & $6 \cdot 1$ & $97 \cdot 1$ & 66 & 3.9 & $4 \cdot 8$ & $6 \cdot 2$ & 1 \\
\hline Vitamin K $(\mu \mathrm{g})$ & $120 \|$ & Regular & 43.8 & 55.4 & 68.4 & 98.4 & 122 & $43 \cdot 8$ & $55 \cdot 3$ & $68 \cdot 1$ & 0 \\
\hline & & Modified & $42 \cdot 8$ & $57 \cdot 3$ & 71.8 & $94 \cdot 3$ & 68 & $42 \cdot 8$ & $57 \cdot 3$ & 71.0 & 0 \\
\hline $\mathrm{Ca}(\mathrm{mg})$ & 10001 & Regular & $639 \cdot 6$ & $742^{\star \star}$ & $919 \cdot 6$ & $82 \cdot 7 \dagger$ & 119 & $681 \cdot 3$ & $829 \cdot 8$ & $1093 \cdot 7$ & 23 \\
\hline & & Modified & 691.6 & 890.4 & $1046 \cdot 0$ & $67 \cdot 1$ & 67 & 673.7 & 891.3 & $1088 \cdot 2$ & 1 \\
\hline $\mathrm{Cu}$ (mg) & 0.7 & Regular & 0.91 & 1.07 & $1 \cdot 22$ & 2.4 & 117 & 0.94 & 1.07 & 1.23 & 1 \\
\hline & & Modified & 0.89 & 1.05 & $1 \cdot 17$ & 1.4 & 67 & 0.89 & 1.05 & $1 \cdot 17$ & 0 \\
\hline $\mathrm{Fe}(\mathrm{mg})$ & 6 & Regular & $9 \cdot 4$ & $11 \cdot 0^{\star}$ & $12 \cdot 5$ & 1.6 & 122 & $9 \cdot 7$ & $11 \cdot 3^{\star}$ & $13 \cdot 6$ & 1 \\
\hline & & Modified & 8.9 & $10 \cdot 2$ & 11.4 & 0.0 & 68 & 9.0 & $10 \cdot 6$ & $12 \cdot 2$ & - \\
\hline $\mathrm{Mg}(\mathrm{mg})$ & 350 & Regular & $220 \cdot 6$ & $258 \cdot 6$ & 295.4 & 98.4 & 120 & $222 \cdot 8$ & $260 \cdot 0$ & $300 \cdot 3$ & 4 \\
\hline & & Modified & 211.5 & 249.0 & 276.4 & 98.6 & 67 & $210 \cdot 9$ & 248.5 & 272.6 & 0 \\
\hline $\mathrm{P}(\mathrm{mg})$ & 580 & Regular & $976 \cdot 7$ & $1098 \cdot 6$ & $1258 \cdot 8$ & $2 \cdot 4$ & 125 & $976 \cdot 7$ & $1098 \cdot 6$ & $1258 \cdot 8$ & 0 \\
\hline & & Modified & $956 \cdot 3$ & 1168.5 & 1371.5 & 1.4 & 70 & $956 \cdot 3$ & 1168.5 & 1371.5 & 0 \\
\hline $\mathrm{K}(\mathrm{mg})$ & 4700|| & Regular & $2110 \cdot 2$ & 2482.7 & 2795.7 & $100 \cdot 0$ & 122 & $2142 \cdot 0$ & $2561 \cdot 2$ & 2851.6 & 0 \\
\hline & & Modified & 2157.5 & 2513.4 & $2749 \cdot 0$ & $100 \cdot 0$ & 68 & $2139 \cdot 6$ & $2501 \cdot 1$ & 2729.8 & 0 \\
\hline $\mathrm{Se}(\mu \mathrm{g})$ & 45 & Regular & 78.8 & $89 \cdot 2^{\star \star}$ & 99.1 & 1.6 & 122 & 79.3 & $89 \cdot 3^{\star \star}$ & 99.5 & 0 \\
\hline & & Modified & 63.6 & 72.4 & 88.6 & 2.9 & 68 & 63.3 & $72 \cdot 4$ & 87.4 & 0 \\
\hline $\mathrm{Na}(\mathrm{mg})$ & $1200 \| \rrbracket$ & Regular & 1989.5 & $2340 \cdot 8^{\star \star}$ & 2602.9 & 1.6 & 125 & 1989.5 & $2340 \cdot 8^{\star *}$ & $2599 \cdot 9$ & 0 \\
\hline & & Modified & $1652 \cdot 6$ & 2064.0 & $2348 \cdot 8$ & 1.4 & 70 & $1652 \cdot 6$ & $2082 \cdot 6$ & $2355 \cdot 3$ & 0 \\
\hline Zn (mg) & $9 \cdot 4$ & Regular & $7 \cdot 3$ & $8 \cdot 3^{\star}$ & 9.4 & $74.8 \dagger$ & 115 & 7.4 & 8.3 & 9.5 & 4 \\
\hline & & Modified & $7 \cdot 7$ & $8 \cdot 6$ & $10 \cdot 0$ & $60 \cdot 0$ & 66 & $7 \cdot 7$ & $8 \cdot 6$ & $10 \cdot 0$ & 1 \\
\hline
\end{tabular}

EAR, Estimated Average Requirement; Q, quartiles; AI, Adequate Intake; RAE, Retinol Activity Equivalent; DFE, Dietary Folate Equivalent; DRI, Dietary Reference Intake.

Median of nutrient intake is significantly different between regular and modified texture diets: ${ }^{\star} P<0.05,{ }^{\star \star} P<0.01$.

Proportion of individuals below the EAR/Al is significantly different between regular and modified texture diets: $\dagger P<0.05, \dagger \dagger P<0.01$.

‡ Food intake only includes food and fluids from meals and snacks, and oral nutritional supplements.

$\S$ Represents sample size with known supplement use where detail on dose was available.

II EAR unavailable, value is the Al for specified nutrient.

II $\mathrm{DRI}$ for males $51-70$ years is different from $>70$ years; $\mathrm{Ca}, \mathrm{EAR}=800 \mathrm{mg}$; $\mathrm{Na}, \mathrm{Al}=1300 \mathrm{mg}$ 
Table 3. Nutrient analysis of food intake only and food intake with micronutrient supplements added for females on regular ( $n$ 297) and modified $(n$ 138) texture diets

\begin{tabular}{|c|c|c|c|c|c|c|c|c|c|c|c|}
\hline \multirow[b]{2}{*}{ Micronutrients } & \multirow[b]{2}{*}{ EAR } & \multirow[b]{2}{*}{$\begin{array}{l}\text { Diet } \\
\text { Texture }\end{array}$} & \multicolumn{4}{|c|}{ Food intake only $\ddagger$} & \multicolumn{5}{|c|}{ Food intake and micronutrient supplements } \\
\hline & & & Q1 & Median & Q3 & $\begin{array}{c}\% \text { of individuals } \\
\text { below EAR/AI }\end{array}$ & $n \S$ & Q1 & Median & Q3 & $\begin{array}{l}\text { Number of individuals } \\
>\text { EAR because of } \\
\text { supplementation }\end{array}$ \\
\hline \multirow[t]{2}{*}{ Vitamin A RAE $(\mu \mathrm{g})$} & 500 & Regular & $492 \cdot 2$ & 604.6 & $728 \cdot 3$ & $27 \cdot 6$ & 290 & $492 \cdot 2$ & $611 \cdot 2$ & $752 \cdot 2$ & 1 \\
\hline & & Modified & $485 \cdot 6$ & $600 \cdot 4$ & $683 \cdot 3$ & $32 \cdot 6$ & 133 & 491.0 & $600 \cdot 6$ & 693.4 & 1 \\
\hline \multirow[t]{2}{*}{ Vitamin $B_{1}(\mathrm{mg})$} & 0.9 & Regular & 1.0 & $1 \cdot 2^{\star *}$ & 1.4 & $13 \cdot 1+\dagger$ & 190 & 1.0 & $1 \cdot 2^{\star \star}$ & 1.4 & 1 \\
\hline & & Modified & 0.9 & 1.0 & 1.3 & 31.2 & 133 & 0.9 & 1.0 & 1.4 & 1 \\
\hline \multirow[t]{2}{*}{ Vitamin $B_{2}(\mathrm{mg})$} & 0.9 & Regular & 1.4 & $1 \cdot 7^{\star \star}$ & $2 \cdot 0$ & $2 \cdot 0+\dagger$ & 288 & 1.4 & $1 \cdot 8^{\star \star}$ & $2 \cdot 1$ & 1 \\
\hline & & Modified & 1.2 & 1.6 & 1.9 & $10 \cdot 9$ & 133 & 1.2 & 1.6 & 1.9 & 1 \\
\hline \multirow[t]{2}{*}{ Niacin (mg) } & 11 & Regular & $19 \cdot 4$ & $21 \cdot 9^{\star \star}$ & 24.5 & $1.0 \dagger \dagger$ & 294 & $19 \cdot 4$ & $21.9^{\star \star}$ & 24.7 & 0 \\
\hline & & Modified & $15 \cdot 5$ & $19 \cdot 7$ & 24.6 & $12 \cdot 3$ & 137 & $16 \cdot 1$ & $19 \cdot 7$ & 24.9 & 0 \\
\hline \multirow[t]{2}{*}{ Vitamin $B_{6}(\mathrm{mg})$} & $1 \cdot 3$ & Regular & 0.9 & $1 \cdot 1$ & $1 \cdot 3$ & 79.5 & 290 & 1.0 & $1 \cdot 1$ & $1 \cdot 3$ & 11 \\
\hline & & Modified & 0.9 & 1.0 & $1 \cdot 3$ & $71 \cdot 7$ & 133 & 0.9 & 1.1 & 1.4 & 3 \\
\hline \multirow[t]{2}{*}{ Folate DFE $(\mu \mathrm{g})$} & 320 & Regular & 199.1 & $239.8^{\star \star}$ & $283 \cdot 6$ & $87.5 \dagger \dagger$ & 289 & $202 \cdot 0$ & $243 \cdot 5^{\star \star}$ & 295.5 & 14 \\
\hline & & Modified & $127 \cdot 9$ & $175 \cdot 3$ & $216 \cdot 2$ & 96.4 & 133 & $128 \cdot 1$ & $175 \cdot 6$ & $220 \cdot 4$ & 5 \\
\hline \multirow[t]{2}{*}{ Vitamin $B_{12}(\mu \mathrm{g})$} & 2 & Regular & 2.5 & 3.1 & 3.8 & 7.7 & 289 & $2 \cdot 7$ & 3.6 & 8.7 & 5 \\
\hline & & Modified & 2.5 & $3 \cdot 2$ & 4.0 & 11.6 & 135 & $2 \cdot 8$ & 3.6 & 8.9 & 4 \\
\hline \multirow[t]{2}{*}{ Vitamin C (mg) } & 60 & Regular & $64 \cdot 0$ & $87.5^{\star \star}$ & $116 \cdot 0$ & $18 \cdot 9$ & 267 & $66 \cdot 4$ & $89 \cdot 6^{\star}$ & $125 \cdot 6$ & 4 \\
\hline & & Modified & $76 \cdot 3$ & $99 \cdot 1$ & $132 \cdot 6$ & $12 \cdot 3$ & 133 & $76 \cdot 8$ & $104 \cdot 0$ & $149 \cdot 4$ & 0 \\
\hline \multirow[t]{2}{*}{ Vitamin D $(\mu \mathrm{g})$} & 10 & Regular & 3.5 & 4.7 & 6.4 & $97 \cdot 6$ & 296 & 7.8 & $30 \cdot 5^{\star \star}$ & 53.5 & 206 \\
\hline & & Modified & 3.8 & $5 \cdot 1$ & $6 \cdot 7$ & 97.8 & 138 & $5 \cdot 1$ & $26 \cdot 7$ & $32 \cdot 0$ & 72 \\
\hline \multirow{2}{*}{$\begin{array}{l}\text { Vitamin E } a- \\
\quad \text { tocopherol (mg) }\end{array}$} & 12 & Regular & $3 \cdot 8$ & 4.5 & $5 \cdot 3$ & $99 \cdot 3$ & 269 & 3.9 & 4.6 & $5 \cdot 7$ & 18 \\
\hline & & Modified & 3.6 & 4.8 & 6.0 & 98.6 & & 3.5 & 4.8 & $6 \cdot 3$ & 5 \\
\hline \multirow[t]{2}{*}{ Vitamin K $(\mu \mathrm{g})$} & $90 \|$ & Regular & 44.4 & $51 \cdot 2^{*}$ & $60 \cdot 7$ & $97 \cdot 0 \dagger \dagger$ & 291 & 44.4 & $51 \cdot 2^{*}$ & $62 \cdot 0$ & 1 \\
\hline & & Modified & 36.5 & $46 \cdot 3$ & $63 \cdot 3$ & 88.4 & 135 & 36.5 & $46 \cdot 2$ & $64 \cdot 2$ & 1 \\
\hline \multirow[t]{2}{*}{$\mathrm{Ca}(\mathrm{mg})$} & 10009 & Regular & 529.1 & 674.2 & 821.5 & $89 \cdot 2$ & 293 & $563 \cdot 6$ & 775.5 & $1071 \cdot 1$ & 66 \\
\hline & & Modified & $524 \cdot 4$ & 657.7 & 881.0 & $89 \cdot 1$ & 133 & $552 \cdot 0$ & $713 \cdot 6$ & 953.4 & 11 \\
\hline \multirow[t]{2}{*}{$\mathrm{Cu}(\mathrm{mg})$} & 0.7 & Regular & 0.77 & 0.88 & 1.02 & $14.8 \dagger \dagger$ & 275 & 0.77 & 0.88 & 1.04 & 1 \\
\hline & & Modified & 0.70 & 0.88 & 1.03 & $25 \cdot 4$ & 130 & 0.71 & 0.90 & 1.07 & 2 \\
\hline \multirow[t]{2}{*}{$\mathrm{Fe}(\mathrm{mg})$} & 5 & Regular & 8.2 & $9.4^{\star *}$ & $10 \cdot 6$ & $0.0 \dagger \dagger$ & 291 & 8.4 & $9 \cdot 6^{\star \star}$ & 11.5 & - \\
\hline & & Modified & $7 \cdot 2$ & 8.6 & $10 \cdot 4$ & 6.5 & 134 & $7 \cdot 2$ & 8.9 & $10 \cdot 8$ & 0 \\
\hline \multirow[t]{2}{*}{$\mathrm{Mg}(\mathrm{mg})$} & 265 & Regular & 184.4 & $214 \cdot 2$ & $242 \cdot 1$ & $85 \cdot 9$ & 290 & 184.4 & $215 \cdot 2$ & 248.5 & 8 \\
\hline & & Modified & $175 \cdot 7$ & $206 \cdot 1$ & $245 \cdot 7$ & 79.7 & 133 & $175 \cdot 7$ & $206 \cdot 4$ & $245 \cdot 7$ & 0 \\
\hline \multirow[t]{2}{*}{$\mathrm{P}(\mathrm{mg})$} & 580 & Regular & $796 \cdot 4$ & 951.9 & $1074 \cdot 2$ & $2 \cdot 7 \dagger$ & 293 & $794 \cdot 2$ & 947.4 & $1078 \cdot 1$ & 0 \\
\hline & & Modified & 768.5 & 932.4 & 1131.9 & 8.0 & 137 & 769.4 & 938.0 & $1145 \cdot 8$ & 0 \\
\hline \multirow[t]{2}{*}{$\mathrm{K}(\mathrm{mg})$} & $4700 \|$ & Regular & 1823.4 & 2113.9 & $2396 \cdot 3$ & $100 \cdot 0$ & 295 & 1823.4 & 2130.8 & $2406 \cdot 5$ & 1 \\
\hline & & Modified & 1798.6 & $2054 \cdot 1$ & $2451 \cdot 8$ & $100 \cdot 0$ & 135 & $1798 \cdot 6$ & $2058 \cdot 2$ & $2502 \cdot 1$ & 0 \\
\hline $\operatorname{Se}(\mu \mathrm{g})$ & 45 & Regular & 63.2 & $70.3^{\star \star}$ & 78.4 & $3.4+\dagger$ & 288 & 63.1 & $70.5^{\star \star}$ & 79.0 & 0 \\
\hline & & Modified & $45 \cdot 6$ & $61 \cdot 2$ & $76 \cdot 1$ & $24 \cdot 6$ & 133 & $46 \cdot 4$ & $61 \cdot 8$ & $77 \cdot 3$ & 1 \\
\hline $\mathrm{Na}(\mathrm{mg})$ & $1200 \| \mathbb{|}$ & Regular & 1745.0 & $2035 \cdot 8^{\star *}$ & 2265.5 & $1.7 \dagger \dagger$ & 296 & $1744 \cdot 3$ & $2035 \cdot 8^{\star *}$ & $2268 \cdot 3$ & 0 \\
\hline & & Modified & 1488.5 & $1766 \cdot 6$ & $2042 \cdot 3$ & $7 \cdot 2$ & 138 & 1488.5 & $1766 \cdot 6$ & $2047 \cdot 4$ & 0 \\
\hline $\mathrm{Zn}(\mathrm{mg})$ & $6 \cdot 8$ & Regular & $5 \cdot 7$ & $6 \cdot 8$ & 7.7 & 49.5 & 269 & $5 \cdot 8$ & 6.9 & 8.1 & 7 \\
\hline & & Modified & $5 \cdot 6$ & 7.0 & 8.4 & $47 \cdot 1$ & 130 & $5 \cdot 8$ & $7 \cdot 2$ & 8.8 & 3 \\
\hline
\end{tabular}

EAR, Estimated Average Requirement; Q, quartiles; Al, Adequate Intake; RAE, Retinol Activity Equivalent; DFE, Dietary Folate Equivalent; DRI, Dietary Reference Intake.

Distribution of nutrient intake is significantly different between regular and modified texture diets: ${ }^{*} P<0.05,{ }^{* *} P<0.01$.

Proportion of individuals below the EAR/Al is significantly different between regular and modified texture diets: $\dagger P<0.05, \dagger \dagger P<0.01$.

‡ Food intake only includes food and fluids from meals and snacks, and oral nutritional supplements.

$\S$ Represents sample size with known supplement use where detail on dose was available.

II EAR unavailable, value is the Al for specified nutrient.

I DRI for females $51-70$ years is different from $>70$ years; $\mathrm{Na}, \mathrm{Al}=1300 \mathrm{mg}$.

males as compared with regular texture consumers. High proportions of male residents had intakes from food/beverages/ ONS below the EAR/AI for regular and modified textures for: vitamin $\mathrm{B}_{6}$, folate, vitamins $\mathrm{D}, \mathrm{E}, \mathrm{K}, \mathrm{Mg}, \mathrm{K}$ and $\mathrm{Zn}$. Vitamin A and $\mathrm{C}$ (for regular texture only) were consumed at levels below the EAR by $>25 \%$ of males. A significantly higher proportion of males on modified texture foods were below the EAR than regular texture consumers for folate. But a significantly higher proportion of males receiving regular texture foods were below the EAR as compared with modified texture consumers for vitamins $\mathrm{C}, \mathrm{D}, \mathrm{Ca}$ and $\mathrm{Zn}$. Intake increased to above the EAR for vitamin D (regular $n$ 83/127; modified texture $n$ 38/70) and Ca (regular texture $n$ 23/127) with micronutrient supplement use.

For females (Table 3), modified texture consumers had significantly lower intakes as compared with regular texture consumers for the following nutrients: vitamin $\mathrm{B}_{1}, \mathrm{~B}_{2}$, niacin, folate, vitamin $\mathrm{K}, \mathrm{Fe}$, Se and $\mathrm{Na}$. Vitamin $\mathrm{C}$ intake was significantly higher for modified texture female consumers as compared with regular texture consumers. High proportions of female residents had intakes from food/beverages/ONS below the EAR/AI for regular and modified textures for: vitamin $\mathrm{B}_{6}$, folate, vitamins D, E, K, Ca, Mg and K. Vitamin A and Zn intakes 
were below the EAR for more than $25 \%$ of female participants for both textures. Vitamin $\mathrm{B}_{1}, \mathrm{Cu}$ and Se intakes were below the EAR for $>25 \%$ of modified texture consumers only. Significantly higher proportions of females on modified texture diets were below the EAR/AI than regular texture consumers for vitamins $\mathrm{B} 1, \mathrm{~B} 2$, niacin, folate, $\mathrm{Cu}, \mathrm{Fe}, \mathrm{P}, \mathrm{Se}$ and $\mathrm{Na}$. Females consuming a regular texture had a higher proportion below the AI as compared with modified texture consumers for vitamin $\mathrm{K}$. Intake increased to above the EAR for vitamin D (regular $n$ 206/297; modified texture $n$ 72/138) and Ca (regular $n$ 66/297; modified texture $n$ 11/138) with micronutrient supplement use.

\section{Discussion}

The M3 study provided an opportunity to examine adequacy of nutrient intake in LTC residents and for the first time, how this is affected by food texture modifications and use of vitamin/ mineral supplements. Poor food intake in LTC is well known, but until now, rigorous evidence to demonstrate the extent of nutrient inadequacy has been limited. Differences between consumption of modified and regular textures were identified; males had fewer differences and four nutrients higher in modified texture consumers, whereas females had several nutrients (8/20) where modified texture consumers ate significantly less than regular texture participants. Similarly, there were more statistically significant differences in the proportion below the EAR/AI for modified texture consumers than regular food consumers for females, with modified textures having higher proportions below the EAR/AI for all but one nutrient. Males had few differences, with modified texture consumers having lower proportions below the EAR/AI for four nutrients, as compared with regular texture food consumers. This difference by sex and texture can be largely explained by the significantly lower energy intake of females consuming a modified texture diet as compared with regular texture female participants. Several nutrients were inadequate $(<\mathrm{EAR})$ or below the AI for both textures for at least $50 \%$ of the participants for both sexes: vitamins $\mathrm{D}, \mathrm{E}, \mathrm{K}, \mathrm{B}_{6}$, folate, $\mathrm{Ca}, \mathrm{Mg}$ and $\mathrm{K}$. Of greatest concern were nutrients where $90 \%$ consumed less than the EAR/AI: vitamin $\mathrm{D}, \mathrm{E}, \mathrm{K}, \mathrm{Mg}$ (males only) and $\mathrm{K}$. Vitamin D micronutrient supplement use improved inadequate intakes for 50$70 \%$ of residents and Ca supplementation improved intake for approximately $20 \%$ of regular texture consumers, but other micronutrient inadequacies were not resolved with micronutrient formulations, due to their infrequent use (online Supplementary Table S1).

A Canadian study ( $n$ 48) conducted in one province compared $3 \mathrm{~d}$ of food record intake of residents in five LTC homes to the $\mathrm{EAR}^{(30)}$. Proportions of males with intakes below the EAR/AI were very similar to the current study with a few exceptions; fewer men in this smaller study were at risk for inadequate intakes of vitamin $\mathrm{A}, \mathrm{E}$ and $\mathrm{Mg}$, whereas more were at risk for folate, $B_{1}, B_{2}$ and $\mathrm{Zn}$ when compared with the $\mathrm{M} 3$ sample. Females in this smaller study had the same or higher proportions below the EAR/AI as compared with the M3 sample, potentially due to the low energy intake of these residents. Noted differences may also be due to more complete nutrient databases used in the M3 study, including foods fortified with folate $^{(30)}$, improvements in menu planning since 2008 and a larger, more diverse sample, where modified textures were analysed separately. In a single Swedish nursing home study $(n 52)^{(10)}$, although the EAR cut-point was not used, the authors note that mean intakes for vitamins D, E, folate and Se were $<60 \%$ of Swedish recommendations at baseline before an intervention, which is in line with the M3 findings on inadequacy for these nutrients. Further, absolute intake values for nine nutrients were consistent with the current study; however, intakes of vitamin $\mathrm{A}, \mathrm{B}_{12}$, $\mathrm{Na}$ and $\mathrm{Ca}$ (females only) were higher in this Swedish sample, whereas mean intakes of vitamin D, E (males only), C, folate (as folic acid), Fe and Se were lower than in the M3 sample ${ }^{(10)}$. Finally, a Finnish study that collected $3 \mathrm{~d}$ of weighed food intake data in twenty-three female residents from the same home identified that food intake generally met recommendations, with vitamin $\mathrm{D}, \mathrm{E}$ and folic acid the only nutrients potentially inadequate, when compared with the Finnish requirements ${ }^{(15)}$. As energy intake was on average lower $(5042 \mathrm{~kJ}(1205 \mathrm{kcal}))$ than the M3 sample, differences are not due to increased intake overall, but may be due to the use of the Finnish requirements which were lower than the DRI for several nutrients. Actual values of nutrients consumed were not provided to make direct comparisons to the M3 sample. Differences in these international studies may also be due to use of different nutrient databases, which are known to vary in quality ${ }^{(31)}$ as well as differences in food supply, micronutrient content of the soil (e.g. Se), fortification practices and menu planning strategies, including use of nutrientdense foods. As all of these smaller studies are almost a decade old, M3 provides updated data on the intake of residents in LTC.

A prior analysis of menus from the M3 study homes, comparing regular and pureed texture menus ${ }^{(32)}$ identified that several nutrients were provided at levels below DRI recommendations for both texture groups (vitamins $\mathrm{B}_{6}$, vitamin $\mathrm{D}$, vitamin $\mathrm{E}$, vitamin $\mathrm{K}$, folate, $\mathrm{Ca}, \mathrm{Mg}, \mathrm{K}$ and $\mathrm{Zn}$ ). Thus, it is not surprising that intake of participants was largely inadequate for many of these nutrients in this study. However, this analysis of M3 data identified that some nutrients were consumed at higher levels for modified texture consumers as compared with regular texture consumers. Although this may seem counterintuitive, it may be explained in two ways. First, as demonstrated in this analysis, modified texture consumers were more likely to consume ONS, which provides not only energy and protein, but also micronutrients. Second, the analysis of menus ${ }^{(32)}$ found that pureed menus generally provided lower amounts of nutrients than regular menus, except for vitamin D, Ca and K. This was attributed to use of pureed recipes that were enhanced with milk, skimmed milk powder or cheese to promote nutrient density ${ }^{(32)}$, explaining the intake differences noted in this analysis. Higher vitamin $\mathrm{C}$ levels for modified textures may be due to the standard of care in some provinces that ensures that final portion size for modified textures are the same as the regular texture (e.g. $1 / 2$ cup of peas $)^{(32)}$; more product needs to be pureed (e.g. $3 / 4$ cup) to result in the same final portion size as the regular texture. Well planned menus that meet the recommended daily allowance are essential to promoting AI of 
residents in $\mathrm{LTC}^{(33)}$. The lower intake for some nutrients by modified texture consumers in this analysis can also be attributed to eating challenges and greater disability and cognitive impairment (Table 1). Even if a menu met the DRI, these challenges would still result in potentially inadequate diets. A prior M3 analysis demonstrated that eating challenges and assistance are important predictors of energy and protein intake when site, staff, dining room and resident-level factors are considered $^{(34)}$. Person-centred care practices, being on a dementia-focused unit, malnutrition and dietitian involvement were also predictive of intake ${ }^{(34)}$. Other research has also noted the multifactorial nature of poor food intake in $\mathrm{LTC}^{(6,35)}$ and thus the need for multifactorial strategies beyond quality and nutrient density of the food provided.

Micronutrients consumed at levels below the EAR may not advance to micronutrient deficiency and the implications of low intake in this population are unclear. Deficiency of a nutrient is dependent on absorption and utilisation, disease states and interaction with medications, and for vitamin $\mathrm{D}$, exposure to the sun $^{(36)}$. Deficiency is best identified through functional changes due to inadequate intake or utilisation of a nutrient, especially as some nutrients have insufficient biomarkers ${ }^{(36)}$. Further, the DRI were designed for healthy individuals to prevent deficiency or diseases associated with deficiency ${ }^{(8,28)}$. For this population of typically frail older adults with various disease conditions, the DRI may not apply. Interventions that demonstrate improved health and functional measures with increased nutrient intake are needed to further understand the implications of low intake in this population.

In addition to use of ONS, there are typically three ways in which nutrient intake can be improved: nutrient-dense food, micronutrient supplement use, or fortification of the nutrient in staple foods. Prior work suggests that use of nutrient-dense ingredients can increase micronutrient content of LTC menus ${ }^{(33)}$, meaning that iatrogenic micronutrient malnutrition due to inadequate intake is not inevitable for most nutrients in this population. In addition to careful menu planning, recipes can be further enhanced with nutrient-dense ingredients to promote intake. For example, energy and protein enhancements have been documented to improve intake ${ }^{(37,38)}$. Micronutrient supplement use for most nutrients occurred in $<15 \%$ of participants in this study, excepting for vitamin D, Ca and $\mathrm{B}_{12}$. With two-thirds to three-quarters of the sample being prescribed vitamin $\mathrm{D}$, it is apparent that guidelines ${ }^{(39)}$ for supplementing this nutrient for LTC residents are resulting in practice change. Vitamin D inadequacy was resolved for a good portion of participants because of this provision of micronutrient pills, whereas relatively few residents consumed $\mathrm{B}_{12}$ at levels less than the EAR without vitamin pill use. Micronutrient supplements may be underutilised in LTC due to concerns for toxicity $^{(40)}$; residents bearing the cost of these formulations as they are not covered by drug benefit programs; persons with dysphagia having challenges with pill consumption; and increased burden placed on care staff during medication administration. Injectable forms of nutrients provided on a monthly or less frequent basis may be a strategy to overcome some of these challenges. Fortification of selected nutrients like vitamin D and Ca in key food products has been demonstrated to be effective ${ }^{(41)}$. However, there are several potential barriers to fortification of food in $\operatorname{LTC}^{(42,43)}$.

\section{Limitations}

Sites were purposively selected and cannot be considered representative of all homes in the four provinces included in the M3 study. Nutrient analysis of food intake is complex and fraught with challenges ${ }^{(44,45)}$ and despite the use of rigorous methods, there are still limitations to this work. To promote efficiency, only estimation was required of side dishes and beverages as well as snacks; the evening snack was noted by LTC staff and the reporting was less accurate. Not all LTC had detailed recipes for modified texture products and some commercial products did not have a full nutrient analysis for all micronutrients considered. Also, LTC staff may not have followed standardised recipes. Nutrient analysis is only as accurate as the database used for foods and ingredients, and errors can occur when converting units and household measures to weights, and assigning weight change factors of raw ingredients due to cooking the food ${ }^{(44,45)}$. Further, databases are known to be inaccurate for some nutrients ${ }^{(46)}$. Finally, micronutrient adjustments for supplement usage may be inaccurate due to poor recording of type of formulation and dosage, as well as known overages and shortages in these products ${ }^{(40)}$. The exclusion of approximately $10 \%$ of vitamin/mineral use as it was injectable or because dose was unavailable is another limitation of this work.

\section{Future work}

This analysis is consistent with prior research that has identified that some but not all nutrients are consumed below recommendations by residents living in LTC. Future work should examine if products that are enhanced with use of nutrientdense ingredients are appealing to LTC consumers and can promote micronutrient intake sufficiently to minimise the proportion of residents with intakes below the EAR/AI. Further, research demonstrating that adequate consumption of micronutrients delays progression or improves conditions linked to key nutrients (e.g. B vitamins and neurological problems), is also needed. This research will also help to fully understand the implications of inadequate intake in LTC residents.

\section{Conclusions}

This analysis of the M3 data from 632 residents living in thirtytwo LTC using weighed and estimated food records for 3 nonconsecutive days, demonstrated that 9/20 micronutrients examined were consumed in amounts below recommendations by a high proportion of participants regardless of sex or texture. Except for vitamin D, micronutrient supplements failed to improve this inadequacy. Consumers of modified texture foods (minced and moist, pureed or liquidised) had lower median intake for some ( males $=4$; females $=8$ ) nutrients, potentially due to eating challenges and low energy intake (for women), as compared with regular food texture consumers. Interventions to target micronutrients that are consumed below 
recommendations, especially those that are low for almost all of the sample (vitamin $\mathrm{D}, \mathrm{E}, \mathrm{K}, \mathrm{Mg}$ and $\mathrm{K}$ ), are required.

\section{Acknowledgements}

The authors thank the research assistants, provincial coordinators, dental hygienists and project managers for their significant contributions to the M3 project. The authors would also like to express the gratitude to the long-term care homes, staff, residents, and families who participated in the M3 study.

The M3 project was funded by the Canadian Institutes of Health Research (Operating Grant 2014 no. 326892). Funders had no involvement in the design, analysis or writing of this article.

The primary author, H. H. K. is the primary investigator for M3 and developed the first draft of the manuscript. J. M. conducted all data analyses and provided tables of results. Coauthors are co-investigators on the $\mathrm{M} 3$ project and reviewed and provided input for this manuscript based on their specific research expertise.

The authors declare that there are no conflicts of interest.

\section{Supplementary material}

For supplementary material/s referred to in this article, please visit https://doi.org/10.1017/S0007114518000107

\section{References}

1. Engelheart S \& Akner G (2015) Dietary intake of energy, nutrients and water in elderly people living at home or in nursing home. J Nutr Health Aging 19, 265-272.

2. Aghdassi E, McArthur M, Liu B, et al. (2007) Dietary intake of elderly living in Toronto long-term care facilities: comparison to the dietary reference intake. Rejuvenation Res 10, 301-310.

3. Rakicioğlu N, Aksoy B, Tamer F, et al. (2016) Nutritional status and eating habits of the institutionalized elderly in Turkey: a follow-up study. J Hum Nutr Diet 29, 185-195.

4. Porter K, Hoey L, Hughes CF, et al. (2016) Causes, consequences and public health implications of low B-vitamin status in ageing. Nutrients $\mathbf{8}, 725$.

5. Zhou J, Huang P, Liu P, et al. (2016) Association of vitamin D deficiency and frailty: A systematic review and meta-analysis. Maturitas 94, 70-76.

6. Bell CL, Lee AS \& Tamura BK (2015) Malnutrition in the nursing home. Curr Opin Clin Nutr Metab Care 18, 17-23.

7. Volkert D (2013) Malnutrition in older adults - urgent need for action: a plea for improving the nutritional situation of older adults. Gerontology 59, 328-333.

8. Institute of Medicine (2003) Dietary Reference Intakes: Applications in Dietary Planning. Washington, DC: National Academies Press.

9. Mila R, Abellana R, Padro L, et al. (2012) High consumption foods and their influence on energy and protein intake in institutionalized older adults. J Nutr Health Aging 16, $115-122$.

10. Lammes E \& Akner G (2006) Repeated assessment of energy and nutrient intake in 52 nursing home residents. $J$ Nutr Health Aging 10, 222-230.

11. Volkert D, Pauly L, Stehle P, et al. (2011) Prevalence of malnutrition in orally and tube-fed elderly nursing home residents in Germany and its relation to health complaints and dietary intake. Gastroenterol Res Pract 2011, 247315.

12. Mila Villarroel R, Abellana Sangra R, Padro Massaguer L, et al. (2012) Assessment of food consumption, energy and protein intake in the meals offered in four Spanish nursing homes. Nutr Hosp 27, 914-921.

13. Nowson CA, Sherwin AJ, McPhee JG, et al. (2003) Energy, protein, calcium, vitamin $\mathrm{D}$ and fibre intakes from meals in residential care establishments in Australia. Asia Pac J Clin Nutr 12, 172-177.

14. Grieger JA \& Nowson CA (2007) Nutrient intake and plate waste from an Australian residential care facility. Eur J Clin Nutr 61, 655-663.

15. Suominem M, Laine T, Routasalo P, et al. (2004) Nutrient content of served food, nutrient intake and nutritional status of residents with dementia in a Finnish nursing home. $J$ Nutr Health Aging 8, 234-238.

16. Rumbak I, Satalić Z, Keser I, et al. (2010) Diet quality in elderly nursing home residents evaluated by Diet Quality Index Revised (DQI-R). Coll Antropol 34, 577-585.

17. Strathmann S, Lesser S, Bai-Habelski J, et al. (2013) Institutional factors associated with nutritional status of residents from 10 German nursing homes (ERNSTES Study). J Nutr Health Aging 17, 271-276.

18. Lopez-Conteras MJ, Zamora-Portero S, Lopez MA, et al. (2010) Dietary intake and iron status of institutionalized elderly people: relationship with different factors. J Nutr Health Aging 14, 816-821.

19. Adolphe JL, Whiting SJ \& Dahl WJ (2009) Vitamin fortification of pureed foods for long-term care residents. Can J Diet Pract Res 70, 143-150.

20. Keller H, Chambers L, Niezgoda H, et al. (2012) Issues associated with the use of modified texture foods. J Nutr Health Aging 16, 195-200.

21. Bannerman E \& McDermott K (2011) Dietary and fluid intakes of older adults in care homes requiring a texture modified diet: the role of snacks. JAMDA 12, 234-239.

22. Keller HH, Carrier N, Slaughter S, et al. (2017) Making the Most of Mealtimes (M3): protocol of a multi-centre cross-sectional study of food intake and its determinants in older adults living in long term care homes. BMC Geriatr 17, 15 .

23. Cichero JA, Steele C, Duivestein J, et al. (2013) The need for international terminology and definitions for texture-modified foods and thickened liquids used in dysphagia management: foundations of a global initiative. Curr Phys Med Rehabil Rep 1, 280-291.

24. Finch H \& Arumugam V (2014) Assessing the accuracy and reliability of direct height measurement for use in adult neurological patients with contractures: a comparison with height from ulna length. J Hum Nut Diet 27, Suppl. 2, S48-S56.

25. Morris JN, Fries BE, Mehr DR, et al. (1994) MDS Cognitive Performance Scale. J Gerontol 49, M174-M182.

26. Morris JN, Fires BE \& Morris SA (1999) Scaling ADLS within the MDS. J Gerontol A Biol Sci Med Sci 11, M546-M553.

27. Watson R \& Deary IJ (1997) Feeding difficulty in elderly patients with dementia: confirmatory factor analysis. Int J Nurs Stud 34, 405-414.

28. Institute of Medicine (2000) Dietary Reference Intakes: Applications in Dietary Assessment. Washington, DC: National Academies Press.

29. National Research Council (1986) Nutrient Adequacy: Assessment Using Food consumption Surveys. Washington, DC: National Academies Press. www.nap.edu/catalog/618/nutri ent-adequacy-assessment-using-food-consumption-surveys 
30. Lengyel CO, Whiting SJ \& Zello GA (2008) Nutrient inadequacies of elderly residents of long-term care facilities in a health district in Saskatchewan. Can J Diet Pract Res 69, 82-88.

31. Finglas PM, Berrry R \& Stley S (2014) Assessing and improving the quality of food composition databases for nutrition and health applications in Europe: The contribution of EuroFIR. Adv Nutr 5, 608S-614S

32. Vucea V, Keller H, Morrison J, et al. (2017) Nutritional quality of regular and pureed menus in Canadian long term care homes: an analysis of the Making the Most of Mealtimes (M3) project. BMC Nutr 3, 80.

33. Lam IT, Keller HH, Duizer L, et al. (2015) Micronutrients on the menu: enhancing the quality of food in long-term care for regular, nontherapeutic menus. Can J Diet Pract Res 76, 86-92.

34. Keller H, Carrier N, Slaughter S, et al. (2017) Prevalence and determinants of poor food intake or residents living in long term care. J Am Med Dir Assoc 18, 941-947.

35. Iuliano S, Olden A \& Woods J. (2013) Meeting the nutritional needs of elderly residents in aged-care: are we doing enough? J Nutr Health Aging 17, 503-508.

36. Ter Borg S, Verlaan S, Hemsworth J, et al. (2015) Micronutrient intakes and potential inadequacies of communitydwelling older adults: a systematic review. Br J Nutr $\mathbf{1 1 3}$, 1195-1206.

37. Castellanos VH, Marra MV \& Johnson P (2009) Enhancement of select foods at breakfast and lunch increases energy intakes of nursing home residents with low meal intakes. J Am Diet Assoc 109, 445-451.
38. Abbott RA, Whear R, Thompson-Coon J, et al. (2013) Effectiveness of mealtime interventions on nutritional outcomes for the elderly living in residential care: a systematic review and meta-analysis. Ageing Res Rev 12, 967-981.

39. Papaioannou A, Santesso N, Morin SN, et al. (2015) Recommendations for preventing fracture in long-term care. CMAJ 187, 1135-1144.

40. Andrews KW, Roseland JM, Gusev PA, et al. (2017) Analytical ingredient content and variability of adult multivitamin/ mineral products: national estimates for the Dietary Supplement Ingredient Database. Am J Clin Nutr 105, 526-539.

41. Lam ITY, Pfisterer K, Duizer L, et al. (2016) Micronutrient food fortification for residential care: a scoping review of current interventions. I Am Med Dir Assoc 17, 588-595.

42. Lam IT, Keller HH, Duizer L, et al. (2015) Nothing ventured, nothing gained. Acceptability testing of micronutrient fortification in long-term care. J Nurs Home Res 1, 18-27.

43. Field KM, Duncan AM, Keller HH, et al. (2017) Effect of micronutrient powder addition on sensory properties of foods for older adults. J Food Sci 82, 2448-2455.

44. Church SM (2015) The importance of food composition data in recipe analysis. Nutr Bull 40, 40-44.

45. Church SM (2009) EuroFIR Synthesis report No 7: food composition explained. Nutr Bull 34, 250-272.

46. Luby CH, Vernon R, Maeda HA, et al. (2015) Lack of correspondence between experimentally determined values of vitamin $\mathrm{E}$ in carrot (Caucus carota L.) and those reported in the USDA National Nutrient Database. HortScience 50, $1595-1597$. 